\title{
Genetics of Amyotrophic Lateral Sclerosis
}

\author{
J. Gorodenker and L.M. Levy
}

ABBREVIATIONS: ALS = amyotrophic lateral sclerosis; UMN = upper motor neuron; LMN = lower motor neuron; fALS = familial amyotrophic lateral sclerosis; sALS = sporadic amyotrophic lateral sclerosis

A myotrophic lateral sclerosis (ALS) reflects a heterogeneous group of neurodegenerative disorders unified by loss of motor neurons in the primary motor cortex, brain stem, and spinal cord, resulting in progressive muscle weakness. Typical ALS (representing $80 \%$ of cases) is a limb-predominant disease characterized by a combination of upper motor neuron (UMN) and lower motor neuron (LMN) symptoms predominantly affecting the extremities. ALS may also present in a bulbar form (20\%), with early symptoms involving muscles innervated by the lower brain stem, affecting articulation, chewing, and swallowing. While the disease may chronically persist in such form, it usually progresses to the generalized muscle weakness of typical ALS. ${ }^{1,2}$

Patients with ALS are more often male and typically present in late middle age. ALS is often rapidly progressive, and most patients die within 3-5 years of onset; however considerable variability exists. ALS is divided into sporadic and familial forms and may be classified by body region of onset, relative mix of UMN and LMN involvement, and rate of progression. Many well-characterized genetic variants exist, and subtypes can vary significantly in usual presentation, including age of onset, rate of progression, and degree of cognitive impairment. ${ }^{1,3}$

The overall incidence of the disease is approximately $1-2$ cases per 100,000 individuals per year, and the prevalence is $4-6$ cases per 100,000 worldwide. ALS is diagnosed clinically by El Escorial (World Federation of Neurology) Criteria, with neuroimaging and neurophysiologic studies predominantly used to exclude other diagnostic entities. ${ }^{4,5}$ Management is largely supportive; however, delay in progression and ventilator dependence has been achieved in selected patients by use of riluzole, an indirect glutamate receptor antagonist and selective blocker of TTX-sensitive sodium channels. ${ }^{1}$

From George Washington University Medical Center, Washington, DC.

Please address correspondence to Joseph Gorodenker, MD, Diagnostic Radiology, George Washington University Hospital, 900 23rd St NW, Washington, DC 20037; e-mail: josephgoro@gmail.com

http://dx.doi.org/10.3174/ajnr.A3831
The phenotypic heterogeneity of ALS presents several difficulties; the diagnosis of ALS is made on clinical grounds and is fundamentally uncertain, ranging from "suspected" to "possible," to "probable" to "definite." There currently is no stated role for neuroimaging to support the diagnosis of ALS; however, diagnosis requires absence of neuroimaging evidence of other disease processes that may explain the observed clinical and electrophysiological signs. ${ }^{4,5}$

Absence of a sensitive and specific test for ALS often results in significant delay of diagnosis, which ranges from 13-18 months from onset of the disease or longer in patients who present with isolated LMN signs. ${ }^{5}$ Furthermore, delayed diagnosis restricts the inclusion of patients in clinical trials and limits early initiation of potential neuroprotective treatments. The variability of ALS progression also limits assessment of potential treatment effects.

TDP-43 is an emergent diagnostic marker of ALS. The presence of TDP-43-positive inclusions in degenerating motor neurons appears to be a specific $(>95 \%)$ albeit modestly sensitive $(<60 \%)$ diagnostic feature of ALS; however, this biomarker remains investigational. The pathologic significance of TDP-43 aggregates is unknown and may reflect misfolding or altered trafficking. ${ }^{6}$

\section{WHAT IS THE PATHOPHYSIOLOGY OF ALS?}

The underlying pathophysiology of ALS is not well understood. Extensive research has outlined several plausible molecular pathways that may contribute to motor neuron degeneration in ALS. These pathways, which continue to be actively investigated, include the roles of oxidative stress and glutamate excitotoxicity, abnormal neurofilament function, protein misfolding, impairment of RNA processing, defects in axonal transport, changes in endosomal trafficking, and mitochondrial dysfunction. ${ }^{3,7}$ Recently, forms of ALS and frontotemporal dementia have been found to share common molecular pathophysiology, a prionlike self-propagating dysregulation in RNA processing, and protein homeostasis. ${ }^{3}$ This highlights the interplay of genetic and environmental interaction in disease initiation and propagation. 


\section{WHAT ARE THE GENETICS OF ALS?}

Most (90\%) of ALS cases are sporadic (sALS), with unknown genetic linkage. The remaining $10 \%$ of cases are familial (fALS), and at least 15 genes have been identified that are implicated in approximately one-third of fALS cases. ${ }^{1,3}$ These may be inherited in an autosomal dominant, autosomal recessive, or X-linked pattern. ${ }^{1}$ These genes include SOD-1 (20\% of fALS cases), C9ORF72 (30\% of fALS cases), TARDBP, FUS, Alsin, FIG-4, SIGMAR1, and $U B Q L N 2$. Each gene is associated with a well-characterized pattern of disease, for example, an Alsin mutation is associated with a slowly progressive disease, with predominantly UMN signs, whereas a mutation in FIG-4, which encodes phosphoinositide5-phosphatase, is associated with a rapidly progressive disease with prominent corticospinal tract signs. An autosomal recessive mutation in SIGMAR1 is associated with a juvenile-onset typical ALS as well as frontotemporal dementia. ${ }^{1,3}$

In contrast, the genetic underpinnings of most sALS cases are unknown, though some of the above genes noted for fALS including SOD1, FUS, C9ORF72, and several others, have been linked to sALS cases as well. These genes may be affected by abnormal copy number, single nucleotide polymorphisms, polyglutamine repeats, and deletion or insertion mutations. Examples include polyglutamine repeats in ATXN2, which codes for Ataxin-2 protein (locus 12q24.12), and SNP associations in APEX1, which codes for Apurinic Endonuclease DNA repair enzyme 1 (locus $14 \mathrm{q} 11.2$ ), a protein that protects cells from the effects of oxidative stress. $^{1,3}$

\section{WHAT IS THE ROLE OF CONVENTIONAL NEUROIMAGING IN ALS?}

Conventional anatomic imaging of the brain and spinal cord is helpful in excluding diseases that may mimic the UMN and LMN signs of ALS. Specifically (per the revised criteria of the World Federation of Neurology Research Group on Motor Neuron Diseases), conventional imaging may be useful in "clinically probable" or "possible ALS." Notably, conventional MR imaging is not required in cases of "clinically definite" disease with a bulbar or a pseudobulbar onset. ${ }^{4,5}$

Differential pathology that may be uncovered includes lesions of multiple sclerosis and cerebrovascular disease, masses, spondylotic or other forms of myelopathy, or radiculopathy. ${ }^{5}$

In patients with ALS, signal intensity changes on proton attenuation, T2-weighted, and FLAIR sequences may be seen anywhere along the cerebrospinal tract, from the centrum semiovale to the brain stem. ${ }^{2,5}$ Typical changes in the brain are often best appreciated on coronal imaging and appear as areas of bilateral symmetric increased signal intensity. ${ }^{8}$ The frequency of cerebrospinal tract lesions in patients with ALS ranges widely across studies (from 15-76\%), with combination imaging approaching a sensitivity above $60 \%{ }^{8}$ Symmetric T2 signal intensity changes have also been described in the anterior temporal subcortical white matter in patients with ALS and dementia. ${ }^{8}$ These lesions correspond to loss of myelin, white matter degeneration, and gliosis.

Other findings noted in patients with ALS include lower whole-brain volume as compared with healthy control subjects, though global brain atrophy is relatively mild and nonspecific, and a characteristic T2 dark rim that is probably related to iron deposition in the precentral cortex (reflecting death of Betz cells) may be present; however, this, too, may be seen in healthy control subjects. $^{5,8} \mathrm{~T} 2$ and T1 hyperintensities of the anterolateral columns of the cervical cord have been described in ALS and may have higher specificity than signal intensity changes on brain MR images. Moreover, such changes have been associated with a younger age at onset and a rapid disease progression. ${ }^{5}$ However, signal changes frequently do not correspond with clinical findings and are overall nonspecific; cerebrospinal tract hyperintensities have been described in healthy subjects and in patients with various other diseases. ${ }^{5}$

Given the overall limitations, the traditional role of conventional structural imaging is to support electrophysiologic studies and exclude alternate diagnoses. ${ }^{2,4}$

\section{IS THERE A ROLE FOR ADVANCED NEUROIMAGING TECHNIQUES IN ALS?}

Advanced neuroimaging techniques, which elaborate on microstructure (structural MRI and DTI), metabolism (hydrogen spectroscopy MR and PET), and neural network integrity (restingstate functional connectivity MRI) have been useful in elucidating the pathophysiology of ALS. These may play a future clinical role but for now remain largely investigational. ${ }^{2}$

High-resolution structural MR imaging allows for detailed analysis of focal atrophy and regional gray-white matter differences and may be combined with computer-aided segmentation, voxel-based morphometry, and surface-based morphometry. ${ }^{2,5,8}$

Current DTI techniques, which evaluate the integrity of white matter tracts, have overall low sensitivity (0.65) and specificity (0.67), and are at present unsuitable for clinical application in the diagnosis of ALS. ${ }^{9}$ Hydrogen spectroscopy MR metrics, which allow for ratio and absolute quantification of metabolites, demonstrate changes across motor neuron diseases; typically decreased NAA, decreased glutamate, and increased choline. These metrics correlate with measures of disease severity and UMN function but are generally nonspecific. ${ }^{2}$ Resting-state functional connectivity MRI and PET studies have demonstrated $\gamma$-Aminobutyric acid system dysfunction in ALS, suggesting a pathophysiologic process possibly mediated by glutamate excitotoxicity. $^{2}$ Of note, advanced machine learning tools have been shown to achieve promising $(>70 \%)$ accuracy for disease state classification by use of these techniques in combination. ${ }^{10}$

\section{REFERENCES}

1. Chen S, Sayana P, Zhang X, et al. Genetics of amyotrophic lateral sclerosis: an update. Mol Neurodegen 2013:8:28

2. Foerster BR, Welsh RC, Feldman EL. 25 years of neuroimaging in amyotrophic lateral sclerosis. Nat Rev Neurol 2013;9:513-24

3. Ling S, Polymenidou M, Cleveland DW. Converging mechanisms in ALS. Neuron 2013;79:416-38

4. Brooks BR, Miller RG, Swash M, et al. El Escorial revisited: revised criteria for the diagnosis of amyotrophic lateral sclerosis. Amyotroph Lateral Scler Other Motor Neuron Disord 2000;1:293-99

5. Agosta F, Chiò A, Cosottini M, et al. The present and the future of neuroimaging in amyotrophic lateral sclerosis. AJNR Am J Neuroradiol 2010;31:1769-77

6. Upadhyayula S, Gearing M, Jonathan Glass J. TDP-43 inclusions in 
the spinal cord: sensitivity and specificity for the diagnosis of sporadic ALS (meeting abstracts). Neurology 2013;80:P02.171

7. Ferraiuolo L, Kirby J, Grierson AJ, et al. Molecular pathways of motor neuron injury in amyotrophic lateral sclerosis. Nat Rev Neurol 2011;7:616-30

8. Kwan JY, Jeong SY, Van Gelderen P, et al. Iron accumulation in deep cortical layers accounts for MRI signal abnormalities in ALS: correlating 7 Tesla MRI and pathology. PLoS One 2012;7:e35241
9. Foerster BR, Dwamena BA, Petrou M, et al. Diagnostic accuracy of diffusion tensor imaging in amyotrophic lateral sclerosis: a systematic review and individual patient data meta-analysis. Acad Radiol 2013;20:1099-100

10. Welsh RC, Jelsone-Swain LM, Foerster BR, et al. The utility of independent component analysis and machine learning in the identification of the amyotrophic lateral sclerosis diseased brain. Front Hum Neurosci 2013;7:251 